Hier können Sie CME-Punkte sammeln a) für die Pflichtfortbildung aller Vertragsärzte und b) für freiwillige Fortbildungszertifikate, die viele Landesärztekammern anbieten.

Die Multiple-Choice-Fragen beziehen sich auf den vorangegangenen Fortbildungsbeitrag (Seiten 50-53). Die Antworten ergeben sich z. T. aus dem Text, z. T. beruhen sie auf medizinischem Basiswissen!
CME-Herausgeber- und Review-Board:

Dr. H. J. K. Barwitz, Prof. Dr. A. Berghaus, Prof. Dr. Dr. h.c. Th. Brandt, Prof. Dr. W. G. Daniel, Prof. Dr. W. Eisenmenger, Prof. Dr. K. Friese, Prof. Dr. H. S. Füessl, Prof. Dr. B. Göke, Prof. Dr. R. Gradinger, Prof. Dr. H. Holzgreve, Prof. Dr. A. Imdahl, Prof. Dr. K.-W. Jauch, Prof. Dr. K. Krüger, Prof. Dr. H.-J. Möller, Prof. Dr. D. Reinhardt, Prof. Dr. Dr. h.c. Th. Ruzicka, Prof. Dr. Ch. Stief, U. Weigeldt.

\title{
Fragen zum Thema: „Therapie der rheumatoiden Arthritis
}

- 1. Pat. 32 J., Schwellungen und Schmerzen der Fingergrundgelenke seit 3 Wochen, Diclofenac lindert den Schmerz, die Schwellungen persistieren. Was tun Sie zuerst?

A Adalimumab alle 2 Wochen $40 \mathrm{mg}$ s. c.

B Methotrexat wöchentlich $15 \mathrm{mg}$

C Dexamethasoninjektion $40 \mathrm{mg}$ i. $\mathrm{m}$.

D vor Einleitung weiterer Therapien zuerst Diagnose sichern

E Krankengymnastik verordnen

2. Was bedeutet der Begriff „Basistherapie" bei der rheumatoiden Arthritis (RA)?

A Diätetische Basismaßnahmen

B Physikalisches Basisprogramm

C NSAR- bzw. Glukokortikoidtherapie zur basalen Schmerzbehandlung

D Krankheitsmodifizierende medikamentöse Langzeitmaßnahmen

E Patientenschulungsprogramm

- 3. Pat. 58 J. RA seit 6 Jahren. Wöchentlich $20 \mathrm{mg}$ MTX, Teilremission. Rezidivierend Kniegelenkserguss. Ihre Maßnahme?

A Gelenkpunktion, Synoviaanalyse, Dexamethason $40 \mathrm{mg}$ i.a.

B MRT des Kniegelenks

C Akupunktur

D MTX auf $40 \mathrm{mg} /$ Woche erhöhen

E Einreibungen mit einem NSAR-Gel

- 4. RA-Pat. seit 2 Jahren erfolgreiche Kombitherapie mit TNF-Blocker und MTX. Im Badeurlaub schwerer „grippaler Infekt“. Ihre Sofortmaßnahmen?

A Bettruhe, Prednisolon, Antibiotikaschutz

B Antibiotika

C TNF-Blocker und MTX absetzen bis zur Rekreation

D Alle "Rheuma“-Medikamente abset- zen, Beginn mit Chloroquin unter Prednisolonschutz

E Einweisung zur intensivmedizinischen Versorgung ins Krankenhaus

- 5. 60-j. Pat., schweres Asthma bronchiale, jetzt RA-Schub. Welches Medikament verordnen Sie zur akuten Beschwerdelinderung?

A Prednisolon, $60 \mathrm{mg}$; $2 / 3$ morgens, $1 / 3$ abends B Etanercept 1 Ampulle i.m.

C Diclofenac $100 \mathrm{mg}$ D Etoricoxib $60 \mathrm{mg}$ E Opiatpflaster

- 6. Die „Number Needed to Treat" hilft bei der Therapiebeurteilung. Was sagt sie aus?

A Die Anzahl der Tagesdosen eines Medikaments in Abhängigkeit von dessen biologischer Halbwertzeit.

B Anzahl der Therapieversuche mit unterschiedlichen Medikamenten, um mindestens einmal erfolgreich zu sein.

C Anzahl der Patienten, die wahrscheinlich von einer ihnen aus Kostengründen vorenthaltenen Therapie profitierten.

D Die Anzahl von Dollars, die gebraucht werden, damit ein Medikament auf den Markt kommen kann.

E Notwendige mittlere Anzahl der zu behandelnden Patienten, wenn mindestens einer von der Therapie profitieren soll.

- 7. Glukokortikoide werden zur Behandlung der RA verwendet. Dabei müssen folgende Regeln eingehalten werden:

A Bei Low-dose-Prednisolontherapie abends die gesamte Dosis einnehmen.

B Dexamethason eignet sich zur akuten Therapie wegen seiner geringen Nebenwirkungen und sollte gespritzt werden.

C Möglichst niedrige Dosis Prednisolon unter Berücksichtigung der Tagesrhythmik des endogenen Kortisons.

D Glukokortikoide festigen in der Langzeittherapie die Wirbelkörper. Durch niedrige Dosen wird die Osteopetrose verhindert.

E Glukokortikoide können MTX oder TNFBlocker als Basistherapeutika ersetzen.

- 8. Bei der symptomatischen Behandlung der RA mit NSAR ist das GI Blutungsrisiko gering, wenn ...

A gleichzeitig Aspirin gegeben wird.

B gleichzeitig Glukokortikoide verabreicht werden.

C ein Coxib anstelle eines traditionellen NSAR eingesetzt wird.

D die Behandelten alt genug sind (> $75 \mathrm{~J}$.), sodass die Magenschleimhaut nicht mehr auf die Noxe reagiert.

E gelegentlich bei dyspeptischen Beschwerden ein PPI eingenommen wird.

- 9. Wodurch lässt sich das erhöhte kardiovaskuläre Risiko bei RA-Patienten senken?

A Bei gesicherter RA grundsätzlich eine Low-Dose-Aspirintherapie einleiten

B tNSAR und Coxibe möglichst niedrig dosieren

C Coxibe grundsätzlich nicht verordnen

D tNSAR bei Patienten mit erhöhtem kardiovakulären Risiko nur zusammen mit PPI verordnen

E Körperlich schonen, bei jeglichen Beschwerden Bettruhe

- 10. 60-j. Frau, seit 4 Wochen fluktuierende Schwellungen der Fingergrundgelenke 2 und 3. Ihre Arbeitsdiagnose?

A Gicht B Rheumatoide Arthritis

C Psoriasisarthritis D Fingerpolyarthrose

E Spondyloarthritis mit peripherer Gelenkbeteiligung

\section{ANTWORTFORMULAR}

Mit Einreichen dieses Fragebogens erkläre ich mich damit einverstanden, dass die angegebenen Daten zum Zweck der Teilnahmebestätigung gespeichert und bei erfolgreicher Teilnahme an den Einheitlichen Informationsverteiler (EIV) der Ärztekammern weitergegeben werden.

Wichtiger Hinweis:

Eine Auswertung ist künftig nur noch möglich, wenn Sie Ihre EFN auf dem Antwortformular angeben! Nähere Hinweise hierzu unter: www.cme-punkt.de/faq.html

\section{"Therapie der rheumatoiden Arthritis“}

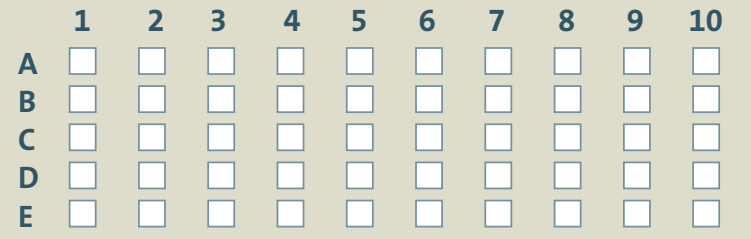

Bitte kreuzen Sie pro Frage je eine Antwort deutlich an.
Name, Vorname

Fachrichtung
Und so kommen Sie zu Ihren Punkten:

Teilnahme im Internet: unter www.cme-punkt.de. Dort führen wir für Sie ein elektronisches Punktekonto.

Teilnahme per Brief: Fragebogen ausfüllen und mit frankiertem Rückumschlag an: Urban \& Vogel GmbH CME MMW-Fortschr. Med. Postfach 81664 München 